\title{
Recul du rivage et montée de la mer sur les plages contrôlées par la houle et sur les plages de poche
}

\author{
BRUNEL, Cédric ${ }^{1}$, SABATIER, François ${ }^{1,2}$ \\ ${ }^{1}$ CEREGE, UMR 6635 CNRS, Centre Européen de Recherche et \\ de l'Environnement, Eurôpole Méditerranéen de l'Arbois, 13545 \\ Aix En Provence Cedex 4, France. Email: brunel@cerege.fr; \\ ${ }^{2}$ Delft University of Technology, Faculty of Civil Engineering, \\ Hydraulic Engineering Section, Stevinweg 1, 2628 CN Delft, The \\ Netherland. Email: sabatier@ cerege.fr
}

Résumé Nous déterminons l'influence de la montée de la mer sur la position du rivage durant le $20^{\text {ème }}$ siècle en Camargue et sur 25 plages de poche de Provence. Il apparaît que la montée du niveau marin est le facteur principal de recul des plages de Provence (60\%), alors qu'elle a un rôle minime dans le recul des plages de Camargue (1.5\%). Des perspectives sont proposées pour 2100 avec l'accélération attendue de la montée de la mer

\begin{abstract}
We firstly determine the importance of the sea level rise on the Camargue and 25 pocket beaches in Provence during the $20^{\text {th }}$ century. The sealevel rise is the major cause of coastal erosion for pocket beaches, whereas it does not represent severe risk for Camargue's beaches. Lastly, based on past changes, and by integrating the geomorphology of the study sites, we evaluate the risks of shoreline retreat linked to a rise in sea level for 2100 .
\end{abstract}

Mots clefs Montée de la mer, érosion côtière, trait de côte.

\section{Introduction}

L'élévation actuelle du niveau marin qui coïncide avec un développement socioéconomique sans précédent des côtes, et sa possible accélération au 20ème siècle en liaison avec le réchauffement climatique ${ }^{[3]}$, est un phénomène qui préoccupe de plus en plus les gestionnaires en charge des espaces littoraux. La vulnérabilité des côtes méditerranéennes face à la montée de la mer a été étudiée par de nombreux auteurs ${ }^{[4]}$, et il est reconnu depuis longtemps que l'élévation marine est un facteur de recul du rivage ${ }^{[2]}$. Notre objectif consiste à quantifier le rôle passé de la montée de la mer dans le recul du rivage ( $20^{\text {ème }}$ siècle), et à proposer une analyse prévisionnelle de la position du trait de côte $\left(21^{\text {ème }}\right.$ siècle) en fonction de la montée de la mer, sur deux types de côtes représentatifs de Méditerranée : les plages de poche de Provence (fig.1), et les plages exposées à la houle du delta du Rhône (fig.2). A priori, ces deux types de côte connaissent une sensibilité différente à la montée de la mer. En effet, les plages du delta du Rhône du fait de leur faible pente, des phénomènes de subsidence ${ }^{[7]}$, et de leur morphologie en lido, semblent particulièrement sensibles à la montée de la mer. A l'opposé, les 
plages de poche de Provence présentent des pentes plus prononcées liées à la présence de sables grossiers. Elles s'organisent en petites unités sédimentaires indépendantes, relativement protégées des tempêtes, et ne connaissent pas de transit littoral significatif du fait de leur position abritée entre les pointes rocheuses qui les délimitent. Les plages de poche du fait de leurs caractéristiques morphodynamiques, semblent donc a priori moins sujettes aux problèmes d'érosion liés à la montée de la mer que les plages de Camargue.

L'analyse des marégraphes de Camargue (Grau de la Dent) et de Marseille (Endoume) réalisée par Suanez et al. ${ }^{[7]}$ a montré qu'entre 1905 et 1993 , ces deux sites sont sensibles à la montée de la mer. En Camargue, la vitesse relative de la montée de la mer $(+2.1 \mathrm{~mm} / \mathrm{an})$ est deux fois plus rapide qu'à Marseille $(+1,1$ $\mathrm{mm} / \mathrm{an}$ ) du fait de la subsidence du delta. Dans cet article, nous utiliserons les données de ces deux marégraphes pour étudier l'impact de la montée de la mer en Camargue et sur les plages de poche de Provence.

Pour déterminer le rôle de la montée de la mer sur la position du rivage, nous comparons la position théorique du rivage, en fonction de la montée de la mer, à celle mesurée, dont la position dépend de la hauteur du plan d'eau mais aussi de la combinaison des dynamiques qui contrôlent l'évolution du rivage (transport longshore, crosshore, apports sédimentaires, processus éoliens ...). La comparaison entre le recul de la ligne de rivage mesuré et le recul calculé en fonction de l'élévation marine, constitue l'originalité de la démarche employée ici : c'est la clef pour quantifier et isoler le facteur " élévation marine » dans la position du rivage.

\section{Présentation des sites étudiés}

Plusieurs dizaines de plages de poche existent le long de la côte rocheuse de Provence (fig.1), entre le delta du Rhône et la frontière italienne, mais seulement 25 d'entre elles répondaient aux critères de sélection choisis pour ce travail : i) existence de données d'évolution du rivage à la fin du XIXème et au XXème siècles, ii) évolution naturelle sans actions anthropiques qui auraient influencé l'évolution du rivage et iii) bassin versant de petite taille afin que les apports fluviaux n'influencent par le budget sédimentaire de la plage. Les plages sélectionnées sont bordées latéralement de pointes rocheuses qui protégent les plages des houles de tempêtes, et s'avancent en mer à une profondeur suffisante (2 à $-10 \mathrm{~m}$ ) pour limiter les entrées et sorties sédimentaires longshore. Le profil des 25 plages de poche est généralement caractérisé par une plage aérienne peu développée (largeur moyenne comprise entre 7 et 30 mètres) et par la présence en arrière de la plage d'une falaise ou d'un talus contre lequel les sédiments de la plage sont adossés. Leur granulométrie est composée de sables moyens à grossiers, ce qui se traduit par des pentes relativement fortes variant de 1 à $3.3 \%$ (moyenne 1.9\%). 


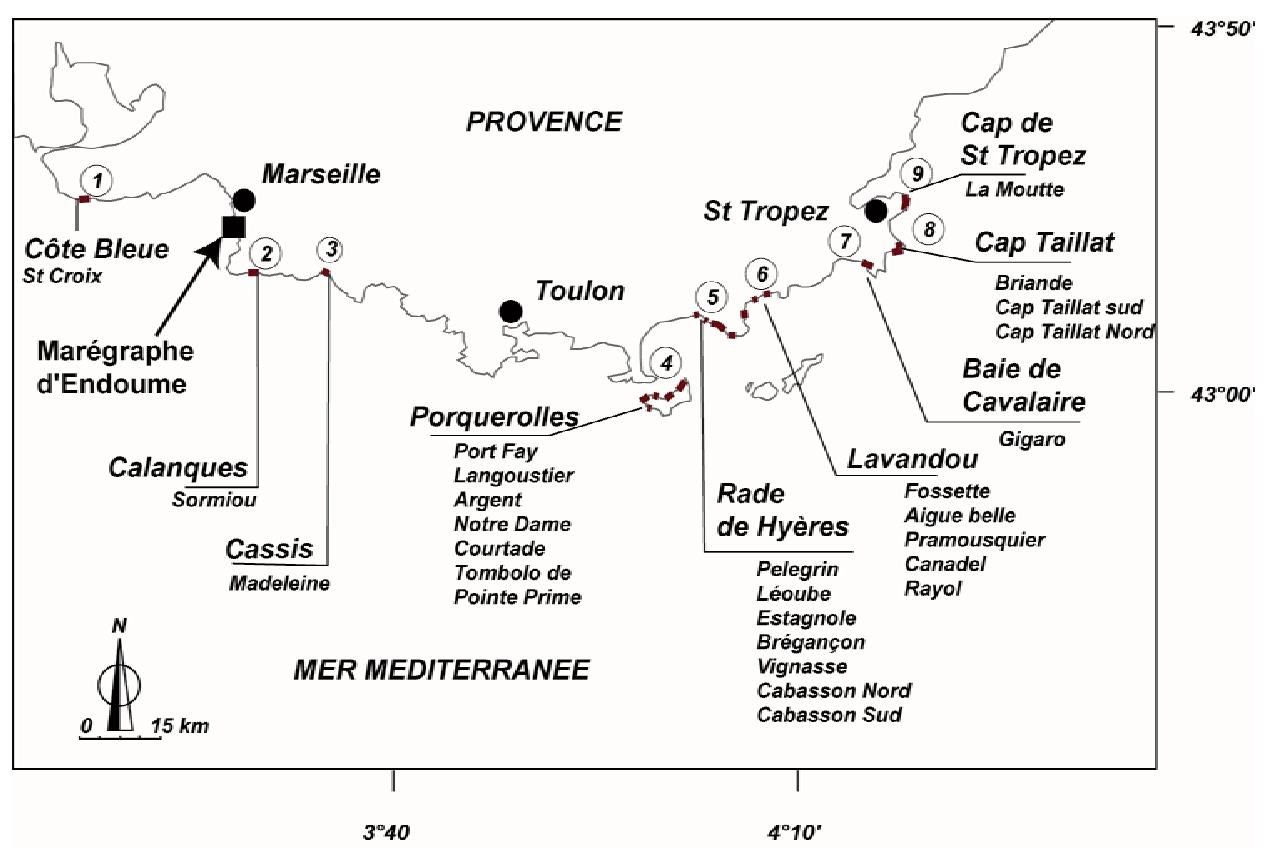

Figure 1 : Localisation des plages de Provence et positionnement du marégraphe d'Endoume.

La frange littorale du delta du Rhône est composée de $90 \mathrm{~km}$ de plages de sable (D50 proche de $0.2 \mathrm{~mm})^{[6]}$, et la ligne de rivage actuelle s'est mise en place au cours du $18^{\text {ème }}$ siècle en s'organisant en cellules littorales ${ }^{[8]}$. La morphologie indique un contrôle important des houles sur la position du rivage ${ }^{[6]}$. Par conséquent, ce type de plage peut être considéré comme un système relativement ouvert, où la houle intervient fortement sur les échanges sédimentaires en comparaison des plages de poche. La pente moyenne des profils de Camargue est plus faible que celle des plages de poche (moyenne $1.7 \%$ ).

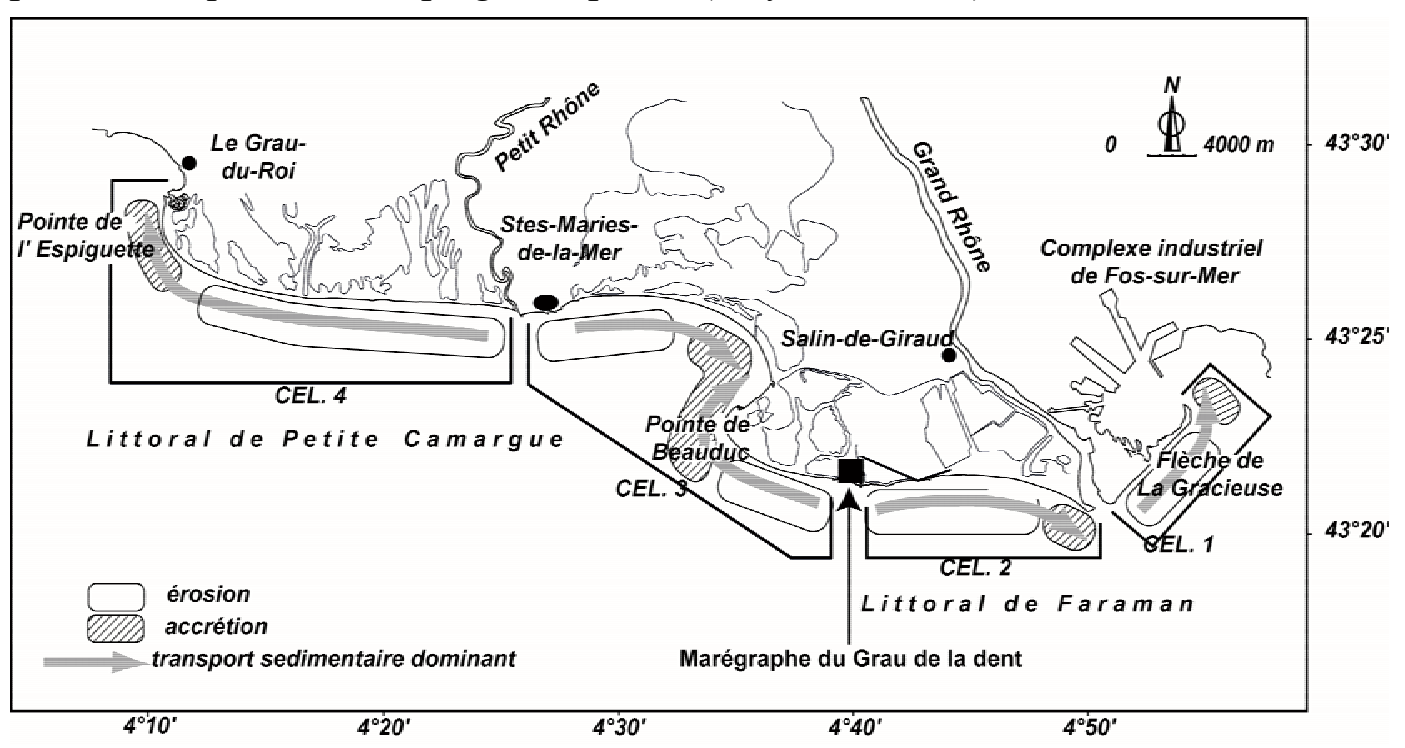

Figure 2 : Localisation des plages de Camargue, distribution des cellules littorales et positionnement du marégraphe du grau de la dent. 


\section{Méthodes et données utilisées}

\section{1. Analyse des données des marégraphes}

Nous utiliserons les marégraphes du Grau de la Dent en Camargue (fig.2), et d'Endoume à Marseille (fig.1), qui nous servirons de référence pour évaluer l'impact de la montée de la mer sur les sites sélectionnés. Cette analyse s'appuie sur celle de Suanez et al. ${ }^{[7]}$, qui ont déjà utilisé les données de ces deux marégraphes, mais dont nous proposons une mise à jour jusqu'en 1998, puisque leur analyse s'arrêtait en 1993.

\section{2. Analyse de l'évolution historique de la ligne de rivage}

Nous avons étudié les variations du rivage durant le $20^{\text {ème }}$ siècle à partir de cartes anciennes (EPSHOM) et des photographies aériennes (IGN) intégrées dans un Système d'Information Géographique (C) MapInfo 6.5). Pour le littoral de Camargue, la période d'étude débute en 1895 et se termine en 1977. Des données récentes existent mais elles ne sont pas prises en compte car à partir des années 80, la ligne de rivage est aussi et surtout contrôlée par les ouvrages de défense côtière. Pour les plages de poche l'étude s'étend de 1895 à 1998, sur des plages qui n'ont pas fait l'objet d'aménagements.

\section{3. Projection de la ligne de rivage par inondation}

Le principe de l'inondation sert de base pour isoler le facteur «montée de la mer» sur le recul du rivage parmi la multitude des agents de forçages qui contrôlent l'érosion côtière. Ce principe s'appuie sur quatre conditions : (1) si on considère que la morphologie du profil de plage est contrôlée par les conditions hydrodynamiques et sédimentaires, et que ces conditions restent constantes, le profil de plage atteint une morphologie stable appelée profil d'équilibre. (2) Si le niveau marin s'élève, et que les conditions hydrosédimentaires restent constantes, la morphologie de la plage retrouve son profil d'équilibre par translation verticale et horizontale du profil. (3) La translation verticale est proportionnelle à l'élévation marine, et la translation horizontale proportionnelle à la pente de la plage ${ }^{[3]}$. Par conséquent, la pente de la plage reste identique, avant et après translation due à l'élévation marine. Plus l'élévation marine est importante, plus le profil va s'élever, plus la pente est douce, plus le profil va reculer, et inversement. (4) Ainsi, si on considère que les pentes du profil de plage restent identiques avant et après élévation marine, il est alors possible d'évaluer le recul de la ligne de rivage, en fonction de deux facteurs : la valeur d'élévation marine et la pente de la plage.

Le principe de l'inondation ressemble finalement au concept du profil d'équilibre utilisé par Bruun ${ }^{[2]}$ dont la loi (Principe de Bruun) est quelques fois remise en cause ${ }^{[1]}$, car les conditions d'application sont difficiles à réunir: équilibre du budget sédimentaire, absence de transit littoral. L'utilisation de ce concept demeure un large débat, il peut être appliqué en première approximation car il peut permettre d'évaluer le rôle de la montée de la mer sur le recul du rivage. Notre approche présente cependant l'avantage majeur de ne pas dépendre 
de ces conditions et s'appuie simplement sur la relation entre montée de la mer et pente de plage. Cette dernière est mesurée entre la position du rivage et la profondeur de fermeture. La principale critique du principe d'inondation réside dans le fait que l'on considère que la pente du profil de plage reste identique durant la période d'étude. Afin de réduire cette approximation, nous utiliserons une pente moyenne mesurée à partir de cartes bathymétriques antérieures à 1977 en Camargue, et des mesures de terrain de 2005 pour les plages de poches. La projection du rivage en fonction de l'élévation marine étant faite par inondation, les résultats sont dépendants des pentes mesurées sur le terrain, et les valeurs de recul obtenues ne sont donc que des valeurs de recul minimum, le recul est potentiellement plus important encore du fait de l'action des autres forçages sur le profil.

\section{Résultats}

\section{1. Rôle de la montée de la mer sur l'évolution de la ligne de rivage durant le 20ème siècle}

Entre 1896 et 1998, les 25 plages de poche étudiées en Provence sont en érosion (fig.3) avec un recul moyen de $-11 \mathrm{~m}$. Le calcul par la technique d'inondation indique un recul moyen de la ligne de rivage de -6,4 mètres entre 1896 et 1998, en considérant une montée de la mer de $11 \mathrm{~cm}$ durant la période. La comparaison entre le recul moyen mesuré $(-0.1 \mathrm{~m} / \mathrm{an})$ et le recul moyen calculé $(-0.06 \mathrm{~m} / \mathrm{an})$ suggère que l'élévation marine joue un rôle important dans le recul du rivage des plages de poche de Provence (environ $60 \%$ en moyenne). Sur certaines plages : Tombolo de la pointe Prime, Pelegrin, La fossette et Pramousquier (fig.1 et fig.3), la montée de la mer pourrait expliquer à elle seule le recul du rivage.

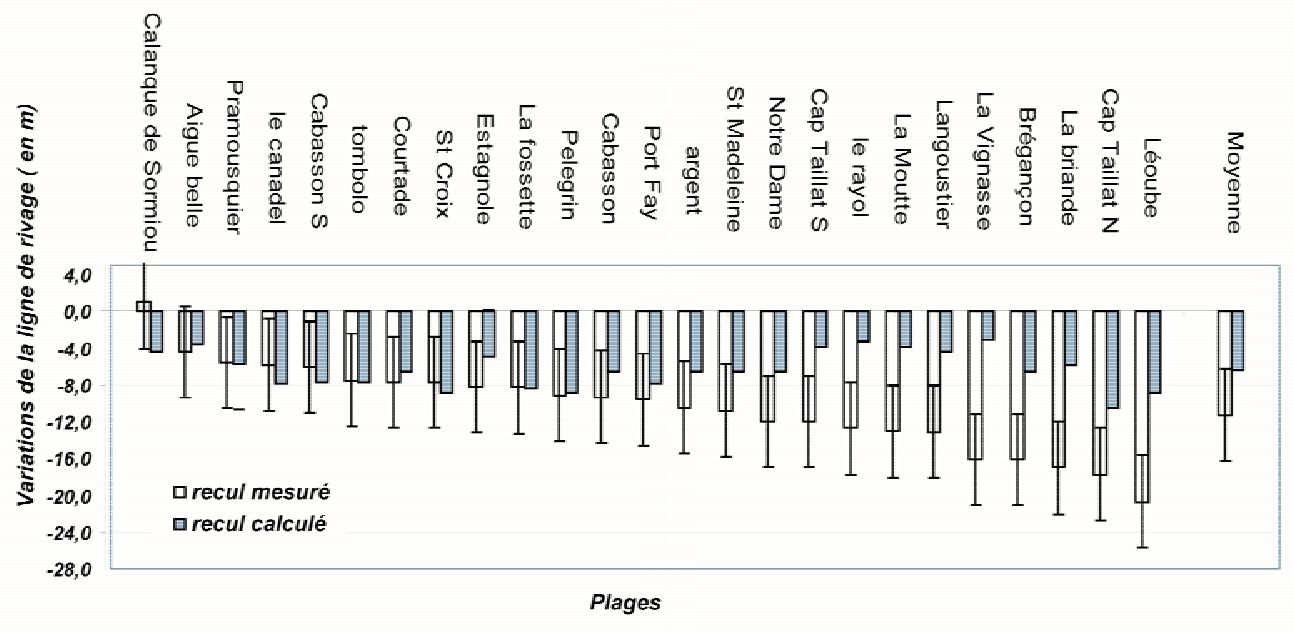

Figure 3 : Comparaison du recul mesuré au recul calculé en fonction de la montée de la mer entre 1896 et 1998 pour les plages de poche de Provence.

(En blanc : recul mesuré ; en gris : recul calculé ; et barres d'erreurs) 
Les variations du trait de côte du delta du Rhône entre 1895 et 1977 (fig. 4A), nous permettent de distinguer des secteurs en accrétion et des secteurs en érosion, confirmant l'organisation en cellules littorales ${ }^{[6]}$.

Les valeurs les plus importantes d'avancée du rivage sont enregistrées à proximité de l'embouchure du Grand Rhône du fait des apports sédimentaires du fleuve, ainsi que sur les flèches sableuses de Beauduc et de l'Espiguette lié au transit littoral (fig.4B) ${ }^{[6]}$.

Les secteurs en recul sont mesurés au niveau des plages de Faraman, des Saintes Maries-de-la-mer et de Petite Camargue (reculs de -250 m à -500 m).

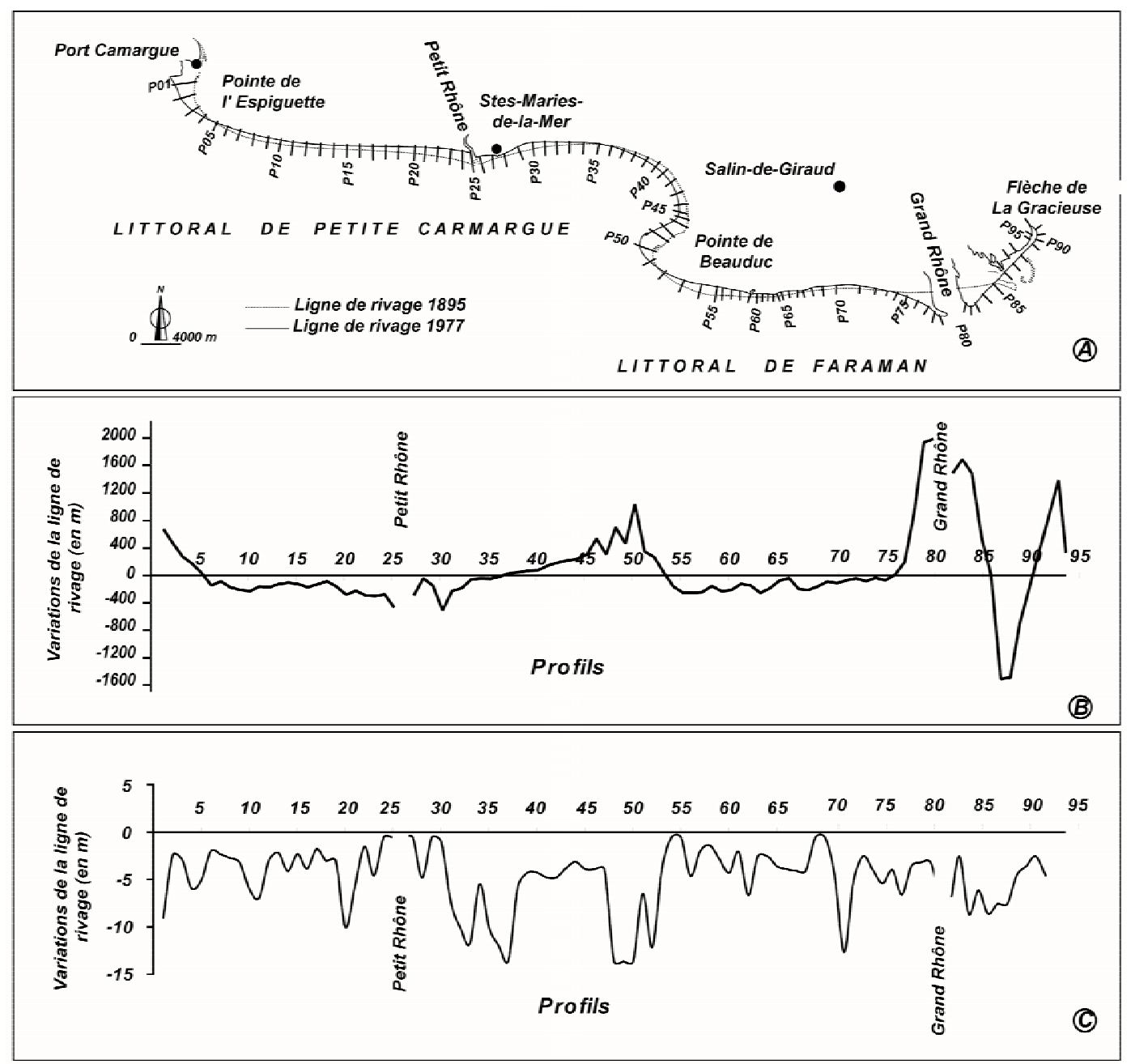

Figure 4 : Variations de la ligne de rivage du delta du Rhône entre 1895 et 1977 . A: Position des profils. B : Evolution mesurée de la ligne de rivage. C : Recul du rivage en relation avec la montée de la mer selon le principe d'inondation.

Sur les zones en érosion, le recul moyen théorique par inondation s'élève à -7.3 m (Fig. 4.C) en considérant une élévation du plan d'eau $17 \mathrm{~cm}$ entre 1895 et 1977. Cette valeur est très largement inférieure au recul mesuré (Fig. 4.B), pour les zones en érosion, le recul moyen mesuré est de $-3.3 \mathrm{~m} /$ an entre 1895 et 1977, alors que le recul moyen calculé en fonction de l'élévation marine est seulement de $0.08 \mathrm{~m} / \mathrm{an}$. Ainsi, le rapport entre les deux indices, montre que la montée de la mer 
serait responsable, en moyenne, de $1.5 \%$ du recul du rivage. Cette valeur doit être considérée comme un ordre de grandeur. Elle témoigne d'un rôle très faible de la montée de la mer sur la position du rivage.

\section{2. Position de la ligne de rivage en 2100}

La projection de la ligne de rivage pour 2100 est réalisée selon le principe de l'inondation en considérant que l'élévation du niveau marin sera de $+4,4 \mathrm{~mm} / \mathrm{an}$ d'ici $2100^{[3]}$. Pour la Camargue, nous avons aussi considéré la subsidence $(+1.1$ $\mathrm{mm} / \mathrm{an})^{[6]}$ et donc une élévation future probable de $+5.5 \mathrm{~mm} / \mathrm{an}$. Cette projection se traduira par un recul moyen dans les zones non aménagées de -23 mètres, variable entre -5 et- 40 mètres. En réalité, il faut s'attendre à un recul plus important car nous venons de démontrer que la montée de la mer joue un rôle modeste dans la position du rivage.

Sur les plages de poche de Provence, l'impact de la montée de la mer sur la position du rivage en 2100 , se traduira par un recul moyen de -25 mètres variant de -12 et -40 mètres. La majorité des plages de poche étant étroites et bordées par un escarpement résistant à l'action des houles, plus haute que la plage et dont la présence limite la translation vers la terre, une partie importante de la surface des plages devrait disparaître suite au recul du rivage. Cette réalité de terrain permet d'établir une classification de la vulnérabilité des plages face à la montée de la mer pour 2100 .

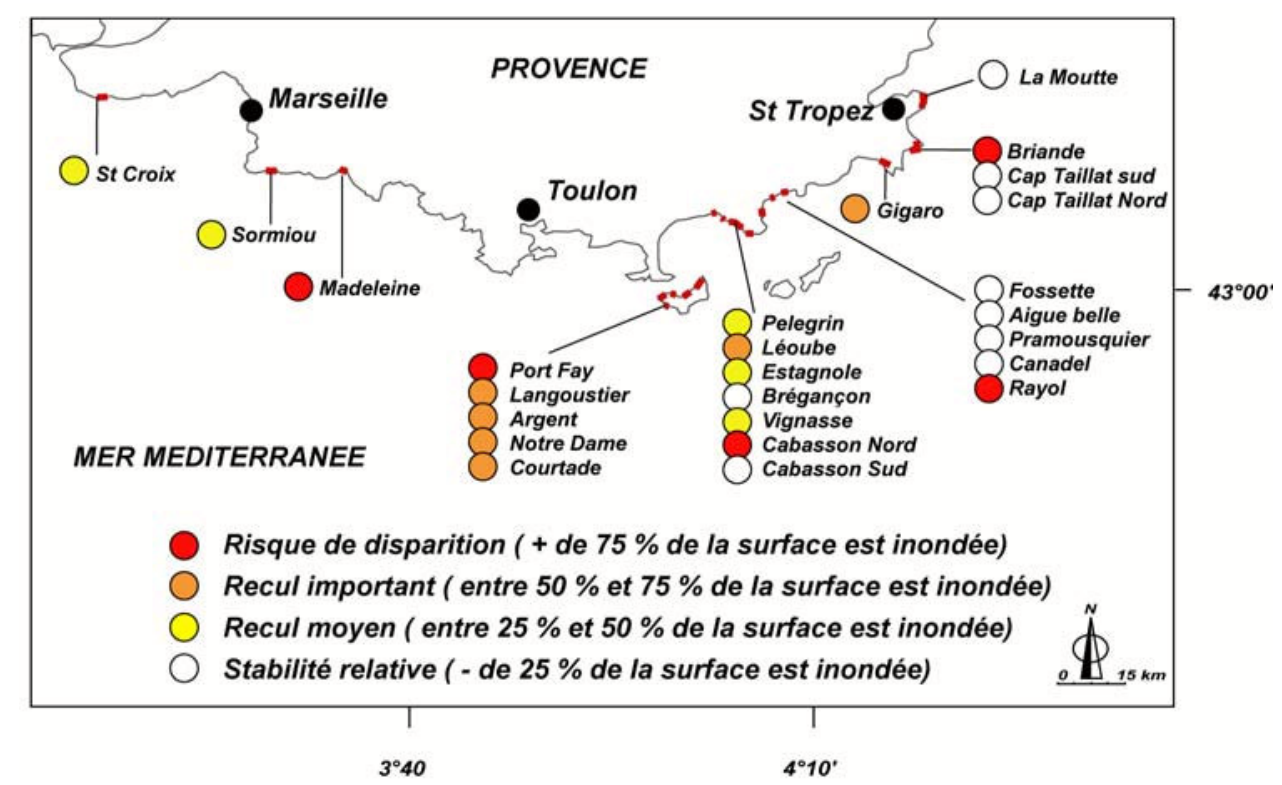

Figure 5 : Vulnérabilité des plages de poche de Provence face à la montée de la mer prévue pour 2100 .

\section{Discussion}

Si l'on considère uniquement la vitesse relative de la montée de la mer sur le long terme, il apparaît que la Camargue est plus sensible à ce paramètre que les plages de poche de Provence. Cependant, l'analyse géomorphologique de ces 
deux types de côtes permet de modérer cette première approximation. En Camargue, l'élévation marine devrait théoriquement induire un recul généralisé du rivage. Cependant, du fait des aménagements et de la redistribution longshore des sédiments, la ligne de rivage ne sera certainement pas positionnée au niveau de l'isoligne $0.55 \mathrm{~cm}$ NGF d'ici une centaine d'années ${ }^{[5]}$. La position du rivage dépendra des ouvrages de protection et des zones en accrétion ou en érosion en relation avec le gradient de transport longshore qui organise les cellules littorales [6]. Par ordre décroissant d'importance, les forçages naturels contrôlant l'évolution de la ligne de rivage sont : (1) le gradient de transport longshore, (2) la faible redistribution des sédiments depuis le Rhône vers les plages, et (3) la montée du niveau marin ${ }^{[5]}$.

Par contre, sur les plages de poche de Provence, si on considère les valeurs de recul à long terme en relation avec la morphologie de l'arrière plage, les plages de poche apparaissent en danger de disparition face à l'élévation marine. Par exemple d'ici 2100, 3/4 des plages de l'île de Porquerolles auront perdu la moitié de leur surface (fig.5).

\section{Conclusion}

Le rôle de la montée de la mer dans l'érosion des plages est variable selon le type de côte. En Camargue, la montée de la mer entre 1896 et 1977 ne représente finalement qu'une proportion réduite du recul total du rivage. En effet, sur les plages exposées à la houle, la montée de la mer est un facteur aggravant mais non prépondérant de l'érosion qui dépend surtout de l'action de la houle et du bilan sédimentaire. Dans l'hypothèse d'une augmentation du nombre des tempêtes, l'influence de la houle dans l'érosion du rivage risque de se maintenir, voire d'augmenter. Autrement dit, la montée de la mer ne constitue pas la cause majeure de l'érosion de ce type de côtes. Par contre, pour les plages de poche protégées de la houle et à l'arrière plage limitée, l'élévation marine est un des principaux facteurs de recul du rivage. L'accélération de la montée de la mer prévue pour 2100 jouera un rôle important dans la position future de la ligne de rivage de ces plages.

\section{References}

1 Andrew, J., Cooper, G., Pilkey, H., (2004). Sea level rise and shoreline retreat: Time to abandon the Bruun Rule. Global and Planetary Change, 43, 157-171.

2 Bruun, P., (1962). Sea-level rise as a cause of shore erosion. Journal of Waterways and Harbors Division, WW1: 117-130.

3 INTERGOVERNMENTAL PANEL ON CLIMATE CHANGE, (2001). Climate change 2001: Impacts, adaptation and vulnerability. -Contribution of the working group to the third assessment report of the intergovernmental Panel of Climate Change, World Meteorological Organization, Genève, 124p.

4 Jeftic, L., Milliman, J.D., Sestini, G., (1992). Climatic change and the Mediterranean: Environmental and societal impacts of climatic change and sealevel rise in the Mediterranean region. Edward Arnold, London, hardcover, XII, $673 \mathrm{pp}$. 
5 Sabatier, F., Provansal, M., and Fleury, T.J., (2005). Discution of : Paskoff, R., 2004. Potential implications of sea-level rise for France. Journal of coastal research, 21, 4, 860,-864.

6 Sabatier, F.and Suanez .S., (2003). Evolution of the Rhône delta coast since the end of the 19th century. Géomorphologie : relief, processus, environnement, 47: 283-300.

7 Suanez, S., Prosper-Laget, V. and Provansal, M., (1997). Variations relatives du niveau marin dans le delta du Rhône et à Marseille, implications tectoniques et/ou climatiques. C. R. Acad. Sci. Paris, 324(IIa): 639-646

8 Vella, C., Fleury, T-J., Raccasi, G., Provansal, M., Sabatier, F., Bourcier, M., (2005). Evolution of the Rhône delta plain in the Holocene. Marine Geology, 222-223, 235-265. 